\title{
What Visual Information Is Processed in the Human Dorsal Stream?
}

\author{
Martin N. Hebart ${ }^{1,3}$ and Guido Hesselmann ${ }^{2}$ \\ ${ }^{1}$ Bernstein Center for Computational Neuroscience and ${ }^{2}$ Visual Perception Laboratory, Department of Psychiatry and Psychotherapy, Charité \\ Universitätsmedizin Berlin, 10117 Berlin, Germany, and ${ }^{3}$ Berlin School of Mind and Brain, Humboldt-Universität zu Berlin, 10117 Berlin, Germany \\ Review of Sakuraba et al.
}

The idea of a division between a dorsal and a ventral visual stream is one of the most basic principles of visual processing in the brain (Milner and Goodale, 1995). The ventral stream originates in primary visual cortex and extends along the ventral surface into the temporal cortex; the dorsal stream also arises in primary visual cortex, but continues along the dorsal surface into parietal cortex. The ventral stream (or "vision-for-perception" pathway) is believed to mainly subserve recognition and discrimination of visual shapes and objects, whereas the dorsal stream (or "vision-for-action" pathway) has been primarily associated with visually guided reaching and grasping based on the moment-to-moment analysis of the spatial location, shape, and orientation of objects. It has been proposed, however, that the dorsal stream also processes tools as a category, so that manipulable objects would be processed by those brain regions that are important for the execution of actions. However, because dorsal and ventral visual regions are heavily interconnected, it is difficult to tell in healthy subjects whether information is processed along the dorsal

Received March 26, 2012; revised April 22, 2012; accepted April 26, 2012. This work was supported by the German Research Foundation (Grant HE 6244/1-1 to G.H.).

Correspondence should be addressed to either of the following: Martin Hebart or Guido Hesselmann at the above addresses. E-mail: martin.hebart@bccn-berlin.de or guido.hesselmann@charite.de.

DOI:10.1523/JNEUROSCI.1462-12.2012

Copyright $\odot 2012$ the authors $\quad 0270-6474 / 12 / 328107-03 \$ 15.00 / 0$ stream only, or whether it is fed to parietal cortex via ventral visual regions.

One way to distinguish between these two alternatives has been offered by the use of continuous flash suppression (CFS) in which noise screens or Mondrian masks flashed at $10 \mathrm{~Hz}$ to one eye suppress the perception of an image presented to the other eye. It has been suggested that this method selectively disrupts ventral visual processing while leaving dorsal processing intact (Fang and He, 2005). In a series of visual priming experiments using CFS, Almeida and colleagues (2008) found that reaction times to visible tools were shorter when they were preceded by masked tools rather than masked animals. No such priming effect appeared when subjects had to react to pictures of animals. Importantly, this result was limited to CFS: category priming using backward masking (in which the stimulus is followed by the mask, typically on both eyes) demonstrated priming effects for both tools and animals. These results, together with previous neuroimaging findings (Fang and He, 2005), were interpreted as evidence that tools, or manipulable objects, are indeed processed as a category directly along the dorsal stream.

A recent paper in The Journal of Neuroscience by Sakuraba and colleagues (2012) addresses an important question left open by this research: if information is already separated into dorsal and ventral streams in early visual cortex, which deals with basic visual features, how is it possi- ble that the visual system can distinguish between manipulable and nonmanipulable objects? Almeida and colleagues (2008) had noted that all tool stimuli used in their experiments had an elongated principle axis, which may explain part of their results. Sakuraba and colleagues (2012) take this idea one step further and explore the processing of elongated shapes and nonelongated tools in more detail. Their results challenge the view that previous findings were specific to the category of tools and add significant new evidence to the question of what attributes of visually presented tools may be processed (presumably in dorsal stream cortical areas) outside of awareness under CFS.

As in the study by Almeida and colleagues (2008), Sakuraba et al. (2012) used CFS with red-green anaglyph glasses and high-contrast random-noise masks to render priming stimuli invisible and analyzed priming effects for different categories of stimuli. For each category, five photographs were prepared; one image was selected as the priming stimulus (shown for $200 \mathrm{~ms}$ ) and the remaining four images in each category served as probe stimuli and were shown until subjects responded to the probe's category (tool or animal). Using this experimental setup, Sakuraba et al. (2012) replicated Almeida's principle finding of a categoryspecific priming effect for tools under CFS.

Next, Sakuraba et al. (2012) presented line drawings instead of photographs as 
priming and probe stimuli and demonstrated that it was not the surface attributes, but only the shape that was driving the tool-specific priming effect. This experiment addressed the critical question of whether the observed priming effects resulted from the category of tools or alternatively from the shape, since many tools have an elongated principle axis. When tools without elongated shape components, e.g., a punch or lemon squeezer, were used as priming stimuli (and standard tool images as probes), category-specific priming effects for tools were no longer observed. As an important variation of this experiment, Sakuraba et al. (2012) presented images of tools in which the tools were shown from noncanonical perspectives that avoided the visual formation of an elongated axis. Again, specific priming effects for tools were absent, further suggesting that the dorsal stream does not process a tool category (Fig. 1).

Nonetheless, many alternative scenarios could account for this null finding. One parsimonious explanation would be that tools without an elongated principle axis and tools depicted in an unconventional view are much harder to recognize and categorize than standard tools. To rule out this possibility, Sakuraba et al. (2012) conducted a third experiment in which thin, elongated rectangles were used as priming stimuli for standard tool images as probes. The response time data showed that these filled rectangles produced significant priming effects similar to the effects observed with line drawings and photographs of tools. One could argue, however, that subjects falsely categorized the elongated rectangles as tools, such as a pencil or simply a stick, because the tool probes, which were visible on each trial throughout the experiment, defined a "tool context". Such false categorization is conceivable for trials in which the priming stimuli remained fully invisible under CFS, and even for trials in which the obliquely oriented rectangle priming stimuli fully or partly broke suppression from random-noise masks; unfortunately, an online, trial-by-trial measure of prime visibility was not recorded for any of the experiments, but the prime contrast was individually adjusted in a control task before the priming procedure to achieve full suppression.

Much stronger evidence against the processing of a tool category in dorsal stream areas is provided by Sakuraba et al.'s (2012) next experiment, in which vegetables were used as priming stimuli

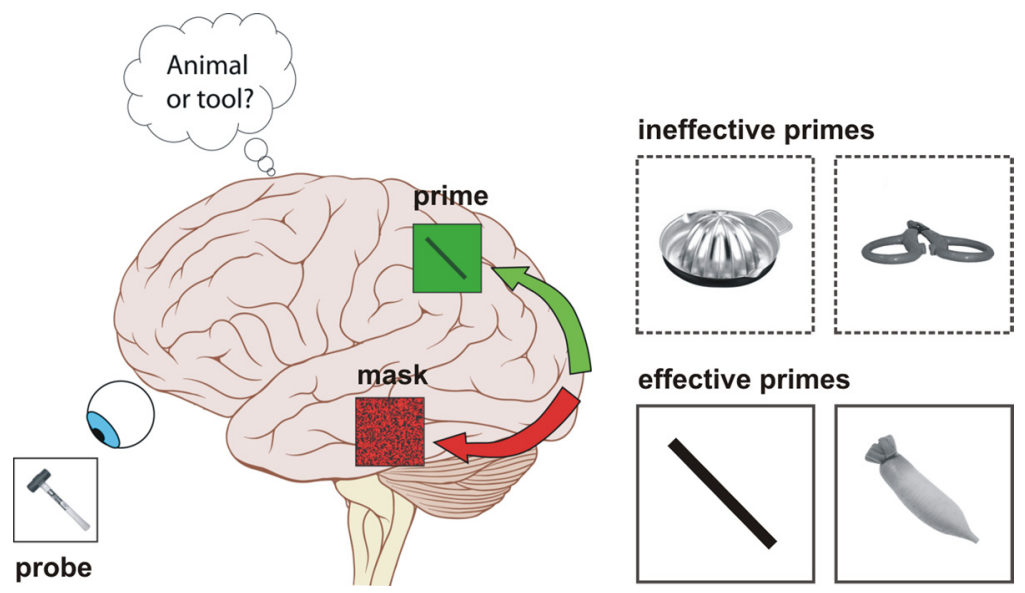

Figure 1. Left, The rationale of the experiments. Continuous flash suppression was used to present masked priming stimuli and specifically target unconscious processing along the dorsal visual stream. After the presentation of the masked priming stimulus, subjects had to categorize a consciously perceived probe stimulus (e.g., hammer) as belonging to the category of tools or animals. Right, Examples of priming stimuli used in the experiments by Sakuraba et al. (2012). Boxes with dashed outlines indicate priming stimuli that failed to produce significant tool priming effects; boxes with solid outlines indicate priming stimuli that produced significant effects. The results demonstrate that the priming effects are related to elongated shapes and are not specific to the category of tools. (Part of the figure was adapted with permission from P. J. Lynch and C. C. Jaffe).

and standard tool images as probes. Surprisingly, robust priming effects were obtained for elongated vegetables with a clear principle axis (such as asparagus or carrot), but not when rounder vegetables (e.g., tomato or onion) were shown as primes. Elongated vegetables produced a priming effect of similar magnitude to that produced by the images of standard tools, ruling out the false-categorization argument and suggesting instead that shape attributes rather than category are indeed the basis of the observed priming effects. Although these results are quite convincing, a critical reader could still argue that the orientation common to pictures of standard tools, elongated rectangles, and elongated vegetables could have produced the same results. Therefore, in their fifth experiment, Sakuraba et al. (2012) presented three types of priming stimuli in three different orientations $\left(0^{\circ}, 90^{\circ}\right.$, and $\left.135^{\circ}\right)$ : elongated rectangles, diamond shapes, and cut circles ("Pac-Man"). Consistent with the shape argument supported by the previous experiments, significant priming effects were found for solid rectangles, but not for diamond or Pac-Man shapes.

Sakuraba et al. (2012) demonstrate a double dissociation between visual processing of tools and elongated shapes. These findings are hard to reconcile with the idea that the human dorsal stream specifically processes a category for tools in the absence of perceptual awareness and thus without input from high-level visual areas in the ventral stream (Almeida et al., 2008). Within the framework of duplex vision, these findings fit nicely into the idea of separate, but heavily interconnected, visual pathways. The results can also be used to reconcile the conflicting results of Fang and He (2005), who demonstrated unconscious processing of tools only in the dorsal stream, and Hesselmann and Malach (2011), who showed stream-invariant responses to tools [see also Kaunitz et al. (2011)]. Possibly, the tools in the former study were more elongated than those in the latter, meaning that low-level differences in stimuli may explain these different results.

These findings can also be embedded in a more general context. Recently, it has been shown that not only dorsal, but also ventral visual areas may exhibit preserved unconscious processing under CFS (Sterzer et al., 2008). Furthermore, behavioral evidence indicates that unconscious priming extends to images that are assumed to be processed along the ventral stream (Barbot and Kouider, 2012). For those reasons, the apparent dissociation between dorsal and ventral visual brain regions brought about by CFS does not seem to hold, and it can therefore be questioned whether the study by Sakuraba et al. (2012) successfully isolated dorsal stream processes. However, because this claim was based on selective unconscious processing of tools (Fang and He, 2005; Almeida et al., 2008), the findings of Sakuraba et al. (2012) demonstrate that such seemingly high-level effects may be explained by basic visual properties such as elongation.

While elongation may be an important dimension for processing along the dorsal visual stream, the presence of a priming 
effect for stick-like figures and for elongated vegetables shows that this can only be part of the story. Indeed, some of the stimuli used to propose the separation of two pathways were nonelongated shapes (Goodale et al., 1994). Future studies should investigate what other aspects of stimuli are processed along the dorsal visual stream. Imaging studies will be crucial to indicate the contribution of ventral visual regions to dorsal stream processing. Sakuraba et al.'s (2012) study is an important first step toward answering the question of which features are important for selective processing in the two visual streams, as demonstrates that low-level stimulus properties may explain behav- ioral responses that are in line with selective activation of high-level brain regions.

\section{References}

Almeida J, Mahon BZ, Nakayama K, Caramazza A (2008) Unconscious processing dissociates along categorical lines. Proc Natl Acad Sci U S A 105:15214-15218.

Barbot A, Kouider S (2012) Longer is not better: nonconscious overstimulation reverses priming influences under interocular suppression. Atten Percept Psychophys 74:174-184.

Fang F, He S (2005) Cortical responses to invisible objects in the human dorsal and ventral pathways. Nat Neurosci 8:1380-1385.

Goodale MA, Meenan JP, Bülthoff HH, Nicolle DA, Murphy KJ, Racicot CI (1994) Separate neural pathways for the visual analysis of object shape in perception and prehension. Curr Biol 4:604-610.
Hesselmann G, Malach R (2011) The link between fMRI-BOLD activation and perceptual awareness is "stream-invariant" in the human visual system. Cereb Cortex 21: 2829-2837.

Kaunitz LN, Kamienkowski JE, Olivetti E, Murphy B, Avesani P, Melcher DP (2011) Intercepting the first pass: rapid categorization is suppressed for unseen stimuli. Front Psychol 2:198.

Milner AD, Goodale MA (1995) The visual brain in action. Oxford: Oxford UP.

Sakuraba S, Sakai S, Yamanaka M, Yokosawa K, Hirayama K (2012) Does the human dorsal stream really process a category for tools? J Neurosci 32:3949-3953.

Sterzer P, Haynes JD, Rees G (2008) Fine-scale activity patterns in high-level visual areas encode the category of invisible objects. J Vis 8:10.1-10.12. 